\title{
Dynamics and Rheology of Actin Solutions
}

\author{
H. I sambert \\ Groupe de Physico-Chimie Théorique, ESPCI, 10 rue Vauquelin, \\ 75231 Paris Cedex 05, France
}

\section{A. C. Maggs*}

ESPCI and Departement of Physics, Princeton University, J adwin Hall, Princeton, New J ersey 08540

Received December 12, 1994; Revised Manuscript Received September 13, $1995^{\circledR}$

\begin{abstract}
We describe the dynamic behavior of semi dilute solutions of actin filaments (a semiflexible polymer), pointing out some of the rheological regimes present in this biologically important system. We deduce the macroscopic storage modulus and show that over a certain frequency range semiflexible polymers show a novel variation in the shear modulus; the mechanism should be distinguished from Rouse behavior in solutions of flexible polymers.
\end{abstract}

\section{Introduction}

Actin is a globular protein of mass $42000 \mathrm{Da}$ which is ubiquitous in the biological world. It is present in an enormous variety of species, from plants to man in a highly conserved form. Within a given species actin is used for a multitude of tasks, both structural and motile. Actin monomers self-assemble under appropriate conditions to form filaments with a diameter of 7 $\mathrm{nm}$ and lengths of up to $50 \mu \mathrm{m}$. The conditions needed for self-assembly can be reproduced in vitro with relatively simple buffers, providing a highly controlled system where the mechanical and thermodynamic properties of filaments can be studied in detail. ${ }^{1}$

Recent experiments using optical microscopy, 2,3 electron microscopy, ${ }^{4}$ and light scattering ${ }^{5-7}$ have shown that for the polymerist actin filaments can be considered as semiflexible polymers with a persistence length of between 10 and $20 \mu \mathrm{m}$. By using additional proteins such as gelsolin, one can control the mean length of filaments present in solution; proteins such as $\alpha$-actinin cross-link filaments to form gels. In addition, with fluorescence marking techniques ${ }^{8,9}$ one can mark and follow a given filament in a rheological experiment, allowing detailed experimental study of reptation dynamics in semidilute solutions. Actin solutions are thus extraordinarily rich systems for rheological studies and pose challenging problems in the study of the dynamics of cellular systems, where control of therheological state of actin plays an essential role in cellular motility. ${ }^{10,11}$

In this paper we discuss the dynamics of actin filaments in semidilute solutions without cross-linking agents and contrast our results with those for classical flexible polymers. The main simplifying assumption we make in this paper is that the effects of polydispersity are weak; in actin solutions we know that due to the process of dynamic polymerization and depolymerization there is in fact a distribution of filament lengths.

In this paper we are particularly interested in explaining three experimentally observed facts: ${ }^{12-15}$

-the value of the plateau modulus observed over a very large frequency range

-the increase in the modulus observed at higher frequencies

\footnotetext{
${ }^{\otimes}$ Abstract published in Advance ACS Abstracts, November 15, 1995.
}

-characteristic time scales for crossovers between the various dynamical regimes

In particular, we note that naive arguments taken directly from the theory of flexible polymers would predict a $\sqrt{\omega}$ variation of the modulus to a Rouselike mode relaxing on scales smaller than the size of the tube. This would imply a crossover from the plateau to a high-frequency regime at about $1 \mathrm{~Hz}$. Experiments show that this crossover occurs rather at $0.01 \mathrm{~Hz}$. We resolve these discrepancies by pointing out the importance of length fluctuations via a longitudinal density mode.

We continue this introduction with a summary of known properties of flexible and semiflexible polymers which will be used later. In section two we introduce a number of length scales which are important in the rheology of actin solutions; in flexible polymers all physics is dominated by the presence of a single length scale; unfortunately, the physics of semiflexible polymers is more complicated, there are rather two main length scales in the problem: the persistence length and the mean distance between filaments. From these two basic length scales we can also construct a number of derived length scales which are important in discussing the rheological behavior. In particular the tube diameter which turns out to be a nontrivial combination of the two basic length scales. We calculate the tube properties and show how they are related to macroscopic properties of actin solutions. In the third section of the article we consider time scales important in actin rheology. Finally, we give numerical estimates of the length and time scales typical in present day experiments on actin solutions.

We start our discussion by a brief reminder of the static and dynamic properties of individual semiflexible polymers together with some remarks on the essential differences between solutions of flexible and semiflexible polymers. The bending energy of a semiflexible polymer is analogous to the bending elasticity of a rigid rod which can be written ${ }^{16}$

$$
E=\frac{\kappa}{2} \int \frac{1}{\mathrm{R}^{2}(\mathrm{~s})} \mathrm{ds}
$$

where $R(s)$ is the radius of curvature of the filament at the point $\mathrm{S}$ and $\kappa$ is the bending el astic constant related to the persistence length $I_{p}=\kappa / k_{B} T$. A strongly curved filament has a small radius of curvature and from (1.1) 


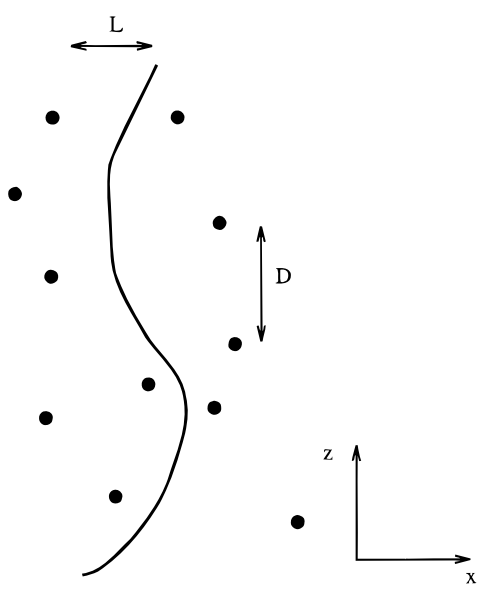

Figure 1. Calculation of the tube diameter. A section of filament of length I is represented undergoing a typical transverse fluctuation of width $L$. The filament is pointing in the $z$ direction and fluctuating in the $x-z$ plane. The cirdes represent filaments in the y direction which act to hinder the fluctuations of the filament. The typical distance between these filaments is D.

has a high energy and is thus energetically unfavorable. On lengths short compared with the persistence length we can use the effective energy

$$
E=\frac{\kappa}{2} \int\left(\frac{d^{2} r_{p}}{d s^{2}}\right)^{2} d s
$$

where $\mathbf{r}_{\mathrm{p}}$ is the two component vector describing the transverse fluctuation of the filament perpendicular to its mean orientation. This energy is to be contrasted with the usual expression for flexible polymers where the energy is related to the first derivative of the position rather than the second.

$$
\mathrm{E}=\frac{\sigma}{2} \int\left(\frac{\mathrm{d} \mathbf{r}}{\mathrm{d} s}\right)^{2} \mathrm{ds}
$$

The dynamics of a free-draining flexible polymer described by eq 1.3 are given by the Rouse dynamic equation:

$$
\xi \frac{\partial \mathbf{r}}{\partial \mathrm{t}}=\sigma \frac{\mathrm{d}^{2} \mathbf{r}}{\mathrm{ds}^{2}}+\eta(\mathrm{t})
$$

with $\sigma$ related to the monomer size. The dynamics of an actin filament on lengths small compared with their persistence length are described by a similar equation with higher spatial derivatives due to the second derivative in the energy (1.2) viz.

$$
\zeta \frac{\partial \mathbf{r}_{\mathrm{p}}}{\partial \mathrm{t}}=-\kappa \frac{\mathrm{d}^{4} \mathbf{r}_{\mathrm{p}}}{\mathrm{ds^{4 }}}+\eta(\mathrm{t})
$$

with $\zeta$ a friction coefficient and $\eta(\mathrm{t})$ Brownian noise. We note that eq 1.5 gives a dispersion relation in $q^{4}$. Throughout this paper we shall neglect small corrections due to nonlocal hydrodynamic effects which give a weak scale dependence to the friction coefficient. Such hydrodynamic effects have been shown to be important, however, in detailed numerical comparisons to lightscattering data. ${ }^{7}$

Simple scaling arguments ${ }^{17}$ (see Figure 1) show that the amplitude of transverse fluctuations, $L$, can be related to the length of a filament, I, in the following way: eq 1.2 is quadratic in the amplitudes $\mathbf{r}_{\mathrm{p}}$; each mode thus has a Gaussian distribution and we may apply equipartition to find

$$
\mathrm{L}^{2} \propto \mathrm{k}_{\mathrm{B}} \mathrm{T} \int_{1 / 1} \frac{1}{\kappa \mathrm{q}^{4}} \mathrm{dq}
$$

Integration of (1.6) implies that

$$
\mathrm{L}^{2} \propto \mathrm{I}^{3} / \mathrm{I}_{\mathrm{p}}
$$

a result wich will be needed later to calculate the tube size. However, we can already see that the scaling between $L$ and I can be the origin of anomalous scaling between the various length scales present in semiflexible polymer solutions. The analogous result for a flexible polymer is that the size of a polymer varies as the square root of the degree of polymerization, a wellknown and classic result. Clearly, eq 1.7 is only valid for length scales smaller than Ip; on lengths comparable with $I_{p}$ we cross over to the description in terms of flexible polymers.

If one confines an object in space there is a restriction of its fluctuations and hence a loss of free energy. In particular the free energy cost of confining a filament to a tube will turn out to be the origin of the plateau modulus in actin solutions. Confining the filament to a tube of diameter $D_{t}$ in which collisions between the filament and the tube occur every $I_{e}$ leads to a free energy cost per unit length comparable to ref 17 .

$$
\mathrm{F}_{\mathrm{c}} \sim \frac{\mathrm{k}_{\mathrm{B}} \mathrm{T}}{\mathrm{l}_{\mathrm{e}}}
$$

Rheological experiments on actin are typically carried out at actin concentrations of the order of $0.3 \mathrm{mg} / \mathrm{mL}$. Under these conditions the filaments of actin form a mesh where the distance between two filaments is between 0.5 and $1 \mu \mathrm{m}$. The rheol ogy of actin filaments differs in a number of respects from the rheology of flexible polymers. ${ }^{18,19}$ In particular the ordering of characteristic length scales with which we are familiar in flexible polymers changes: In flexible polymers the persistence length is often only a few monomers (or tens of angstroms) long; this length is small compared with the correlation length for density fluctuations $\xi$; the correlation length is in turn small compared with the length, $\mathrm{N}_{\mathrm{e}} \xi$, needed to produce entanglements, which determines such macroscopically important quantities as the plateau modulus via the equation

$$
\mathrm{G}_{\mathrm{flex}} \approx \frac{\mathrm{k}_{\mathrm{B}} \mathrm{T}}{\xi^{3} \mathrm{~N}_{\mathrm{e}}}
$$

where $\mathrm{N}_{\mathrm{e}}$ is the number of segments of size $\xi$ needed to form a topological entanglement. Under the usual experimental conditions the persistence length of actin filaments is much larger than the typical mesh size; it also turns out to be much larger than the characteristic length which determines macroscopic elastic properties. This necessitates a reconsideration of the rheology of semiflexible polymers. We note that similar considerations are needed in the rheology of polyelectrolytic solutions where the persistence length is equal to the correlation length. 20

\section{Statics of Actin Solutions}

We now turn to the calculation of the length scales important in actin solutions, together with estimates of the plateau modulus. Long linear filaments formed 
out of monomers with concentration c form a mesh with a characteristic length scale D where

$$
\mathrm{D} \sim(\mathrm{ca})^{-1 / 2}
$$

with a the size of the protein molecules.

We start with a free volume calculation of the tube diameter and the distance between collisions between a filament and the tube wall. The fluctuations of a given filament will be most hindered by nearby filaments which are nearly perpendicular (see Figure 1). These nearby filaments form an array of steric hindrances separated by $D$. Let us consider a filament aligned in the $z$ direction and consider fluctuations of our filament in the $x$ direction. The fluctuations are hindered by filaments in the $y$ direction. The filaments in the $y$ direction create a series of cells of area $D^{2}$. The transverse fluctuations described in (1.7) imply that a segment of filament of length I has fluctuations in the $\mathrm{x}-\mathrm{z}$ plane with area

$$
\mathrm{A} \approx \mathrm{LI} \approx \frac{\mathrm{I}^{5 / 2}}{\mathrm{I}_{\mathrm{p}}^{1 / 2}}
$$

Thus we expect that fluctuations of filaments are hindered when $D^{2} / A \approx 1$, implying that the filament is effectively enclosed in a tube of diameter

$$
D_{t} \sim D^{6 / 5} / /_{p}^{1 / 5}
$$

with points of steric hindrance separated by distances scaling as ${ }^{21}$

$$
\mathrm{I}_{\mathrm{e}} \sim \mathrm{D}^{4 / 5} \mathrm{I}_{\mathrm{p}}^{1 / 5}
$$

We note that there is no reason to consider these points of collision as being entanglements (see below). From these basic length scales we can deduce the macroscopic plateau modulus using the following arguments: The plateau modulus is due to stresses in the medium which can only be released by reptation of the filament out of the tube. Rather than calculating stress we shall calculate the energy scales implicated in deformations of order 1 . For concreteness we shall consider deformations of the form

$$
\left(\begin{array}{lll}
1 & \epsilon & 0 \\
\epsilon & 1 & 0 \\
0 & 0 & 1
\end{array}\right)
$$

which correspond to a stretching of the system in the direction $(1,1,0)$ and a compression in the direction (1, $-1,0)$. Under such a deformation the tube which contains each filament is sheared and is thus no longer described by its original Gaussian distribution in space. Each section of the tube of length $I_{p}$ will then contribute an additional $k_{B} T$ to the free energy of the filaments. This implies that the contribution to the modulus, due to the change in the conformation of the tube, is equal to $G_{\text {pers }} \sim \mathrm{k}_{\mathrm{B}} T / \mathrm{p}_{\mathrm{p}} \mathrm{D}^{2}$. However, this contribution to the modulus is small compared to the experimentally observed value; there is a second more important contribution.

We know that in flexible polymers the concept of entanglement, expressed via the parameter $N_{e}$, is crucial in defining the idea of tube confinement. ${ }^{18}$ This allows us then to understand important rheological properties, like the plateau modulus of a polymer solution. No entanglement is required to define the confining tube for actin solutions in the regime where
$D \ll I_{p}$. Looking at the typical geometry sketched in Figure 1, one can well believe that filaments are free to slide with respect to each other and that entanglements must occur on the scale of the persistence length rather than the mesh size.

Cross-linking impurities present in very low concentrations would be sufficient to significantly modify the elastic behavior by formation of a reversible or irreversible gel (according to the impurity present), forming a far stiffer macroscopic system; indeed, impurities present at the level of a few parts per thousand would be enough to invalidate the following discussions. We note that fluorescence measurements indicating free movement of filaments 8,9 seem to indicate that there is no crosslinking occurring in typical preparations, and we ignore the possibility in what follows; however, the enormous variability in reported moduli may be at least partially due to cross-linking impurities.

The structure of the tube is far from uniform; it is formed from other pairs of filaments separated by a distance of order $\mathrm{I}_{\mathrm{e}}$. Under the influence of the transformation (2.5) this distance increases by a factor $(1+$ $\epsilon)$ for tubes parallel to $(1,1,0)$ and decreases by a factor $(1-\epsilon)$ for tubes in the direction $(1,-1,0)$. In addition, tubes in the direction $(0,0,1)$ are distorted in shape. Since each section of tube of length $\mathrm{I}_{\mathrm{e}}$ contributes $\mathrm{k}_{\mathrm{B}} T$ to the free energy of the solution, we might expect that the nonuniformity of the tube leads to anisotropic variations in $\mathrm{I}_{\mathrm{e}}$ and hence from (1.8) variations in $\mathrm{F}_{\mathrm{c}}$. This mechanism would be expected to contribute

$$
\mathrm{G}_{\mathrm{pl}} \sim \frac{\mathrm{k}_{\mathrm{B}} \mathrm{T}}{\mathrm{I}_{\mathrm{e}} \mathrm{D}^{2}}
$$

to the plateau modulus. We believe that this is the dominant contribution to the plateau modulus and is a major result of this paper.

The discussion leading to the result (2.6) is valid on time scales sufficiently long that the internal modes of the filament do not contribute to the rheol ogical behavior. We now consider the modulus at higher frequencies, in particular at the frequency of relaxation of a section of the filament of length $I_{e}$. (We give an explicit formula for this time, $\tau_{\mathrm{e}}$, later in the paper in the discussion of dynamic effects.) On this time scale the basic mechanical element is the portion of filament of length $I_{e}$ between each collision point.

This element can be described in terms of transverse and longitudinal elastic constants such that the energy, $\mathrm{E}$, of a fluctuation is related to a displacement $\delta \mathrm{r}$ via $\mathrm{E}$ $=\mathrm{K}(\delta \mathrm{r})^{2} / 2$, where $\mathrm{K}$ is a Hookean spring constant or modulus. The modulus describing transverse fluctuations follows easily from eq 1.2; more detailed calculation is needed for the longitudinal displacements: The longitudinal modulus has as its origin the "uncrumpling" of the thermal fluctuations due to an external force. It is thus entropic in origin, while the transverse modulus is enthalpic.

The longitudinal constant can be calculated in a simple Gaussian approximation such as eq 1.2 by calculating the moments of the fluctuation in length of the filament, $\delta \mathrm{s}$, using the following approximation for the excess material in a segment of the tube

$$
\delta \mathrm{s} \approx \frac{1}{2} \int\left(\nabla \mathbf{r}_{\mathrm{p}}\right)^{2} \mathrm{ds}
$$

The longitudinal and transverse elastic constants do not scale in the same manner with the persistence length. 
A section of filament of length I can be considered as an anisotropic Hookean spring with Iongitudinal modulus

$$
\mathrm{K}_{\mathrm{I}} \approx \frac{\mathrm{K}_{\mathrm{B}} \mathrm{TI}_{\mathrm{p}}^{2}}{\mathrm{I}^{4}}
$$

and transverse modulus

$$
\mathrm{K}_{\mathrm{t}} \approx \frac{\mathrm{k}_{\mathrm{B}} \mathrm{TI}_{\mathrm{p}}}{\mathrm{I}^{3}}
$$

We now discuss the statistics of fluctuations of the filament in the tube. From eq 2.8a the mean square fluctuation of the quantity of material in a section of tube of length $\mathrm{I}_{\mathrm{e}}$ is given by

$$
(\delta \mathrm{s})^{2}=\frac{\mathrm{l}_{\mathrm{e}}^{4}}{\mathrm{I}_{\mathrm{p}}^{2}}
$$

Each successive section of filament of length $\mathrm{I}_{\mathrm{e}}$ fluctuates independently, thus the fluctuation in material in a section of tube of length $I>I_{e}$ is

$$
(\delta s)^{2}=\frac{\mathrm{I}_{\mathrm{e}}{ }^{4}}{\mathrm{I}_{\mathrm{p}}{ }^{2}} \frac{\mathrm{I}}{\mathrm{I}_{\mathrm{e}}}
$$

From (2.10) we also see that the process of tube renewal involves lengths of filaments which are long compared with the persistence length. It is probable that in most experimental systems the whole filament is the dynamic object involved in tube renewel.

\section{Dynamics of Actin Solutions}

In this section we describe the various time scales associated with the dynamics of actin filaments. The dynamics described by eq 1.4 are valid until the wave vector q is comparable with the smallest length scale of the actin network, D. Thus, we expect that hydrodynamic interactions between filaments and their environment become important for

$$
\tau_{\mathrm{D}} \approx \beta \zeta \frac{\mathrm{D}^{4}}{\mathrm{I}_{\mathrm{p}}}
$$

with $\beta=1 / k_{\mathrm{B}} \mathrm{T}$. For times shorter than $\tau_{\mathrm{D}}$ the rheology is dominated by the coupling of the bending modes with the shear and the fact that the classical result for independent semiflexible polymers must hold, ${ }^{24}$ viz. $\mathrm{G}(\omega) \approx \omega^{1 / 4}$. However, in actin this result holds only for frequencies higher than $100 \mathrm{~Hz}$, a frequency range which is as yet unstudied. For times longer than $\tau_{\mathrm{D}}$ the hydrodynamic interaction between the tube and filament becomes important, thus increasing the effective friction coefficient $\zeta$. The variation in $\zeta$ could be important; however in this simple scaling analysis we shall not distinguish in what follows between the effective friction coefficient at times which are long or short compared with $\tau_{\mathrm{D}}$. For times somewhat longer than $\tau_{\mathrm{D}}$ the dynamics are thus still described by the $\mathrm{q}^{4}$ dispersion relation implied by eq 1.5 .

The first real change of dynamic regime comes when we consider excitations of wavelengths comparable to the length, $I_{\mathrm{e}}$. At this scale the movement of the filament becomes sterically hindered by the presence of other filaments. This defines the second important time scale in the dynamics

$$
\tau_{\mathrm{e}}=\beta \zeta \frac{\mathrm{D}^{16 / 5}}{\mathrm{I}_{\mathrm{p}}^{1 / 5}}
$$

We note that this time scale is only very weakly dependent upon the rigidity of the filament.
On length scales larger than $I_{e}$ the nature of the dynamics changes from transverse to longitudinal. Fluctuations of amplitude larger than $D_{t}$ are impossible due to the presence of the tube. However, as we have noted, there is a fluctuation in the quantity of material present in each segment of tube of length $I_{e}$; these fluctuations in density diffuse along the tube path. Thus the effective dispersion relation changes from $\mathrm{i} \beta \zeta \omega$ $=I_{p} q^{4}$ to

$$
\mathrm{i} \omega \approx \frac{\left(\mathrm{ql}_{\mathrm{e}}\right)^{2}}{\tau_{\mathrm{e}}} \quad \mathrm{ql}_{\mathrm{e}}<1
$$

We are thus in a position to calculate the time needed to relax fluctuations of a section of filament of length $I_{p}$

$$
\tau_{\mathrm{p}} \approx\left(\frac{\mathrm{I}_{\mathrm{p}}}{\mathrm{I}_{\mathrm{e}}}\right)^{2} \tau_{\mathrm{e}} \approx \beta \xi \mathrm{I}_{\mathrm{p}}^{7 / 5} \mathrm{D}^{8 / 5}
$$

The density fluctuations in the whole tube relax in a time equal to

$$
\tau_{\text {relax }} \approx \beta \zeta \mathrm{L}_{\mathrm{f}}^{2} \mathrm{I}_{\mathrm{p}}{ }^{-3 / 5} \mathrm{D}^{8 / 5}
$$

Between the time scales $\tau_{\mathrm{e}}$ and $\tau_{\mathrm{p}}$ rheol ogical dynamics are dominated by diffusive propagation of density fluctuations al ong a linear tube. These density fluctuations are strongly coupled to the shear flow. Density fluctuations on scales longer than $I_{p}$ are, however, weakly coupled to the shear flow. Thus we expect the crossover to plateau modulus at a time $\tau_{\mathrm{p}}$.

This result should be contrasted with that predicted by a Rouse mechanism. In the Rouse dynamics of flexible solutions we find a rheological regime in $\sqrt{\omega}$; however the crossover from high frequency to plateau behavior occurs at the equivalent of $\tau_{\mathrm{e}}$. This is because the shear flow does not couple to length fluctuations for a highly coiled polymer in linear rheology; for semiflexible polymers there is an important coupling even in the linear regime.

The plateau modulus given by eq 2.7 is valid from the time $\tau_{\mathrm{p}}$ until the moment that filament is able to diffuse completely out of the tube. This defines the longest characteristic time in the problem, which is independent of the actin concentration 25,26

$$
\tau_{\text {rept }} \approx \beta \xi \mathrm{L}_{\mathrm{f}}^{3}
$$

Beyond this time scale the solution is fluid.

\section{Length and Time Scales}

We wish to give here approximate numerical estimates of the fundamental lengths and times which are important in the dynamics of semidilute actin solutions. Typical experiments are performed at concentrations of $0.3 \mathrm{mg} / \mathrm{mL}$, which implies the following length scales

$$
\begin{gathered}
\mathrm{D}=0.6 \mu \mathrm{m} \\
\mathrm{D}_{\mathrm{t}}=0.4 \mu \mathrm{m} \\
\mathrm{I}_{\mathrm{e}}=1 \mu \mathrm{m} \\
\mathrm{I}_{\mathrm{p}}=15 \mu \mathrm{m} \\
\mathrm{L}_{\mathrm{f}}=30 \mu \mathrm{m}
\end{gathered}
$$

$L_{f}$ must be considered as a rough estimate since it is difficult to determine the length of filaments in unmarked samples. From the equations of section three 
we thus estimate that the relevant time scales for the dynamics are

$$
\begin{gathered}
\tau_{\mathrm{D}} \approx 0.02 \mathrm{~s} \\
\tau_{\mathrm{e}} \approx 0.4 \mathrm{~s} \\
\tau_{\mathrm{p}} \approx 100 \mathrm{~s} \\
\tau_{\text {relax }} \approx 400 \mathrm{~s} \\
\tau_{\text {rept }} \approx 10000 \mathrm{~s}
\end{gathered}
$$

It should be noted that the time scales $\tau_{\mathrm{D}}, \tau_{\mathrm{e}}$, and $\tau_{\text {relax }}$ are strongly decreasing functions of the concentration. We can compare these time scales with those seen in recent experiments..$^{13}$ Over a large frequency range from 0.01 to $0.0001 \mathrm{~Hz}$ one observes a plateau modulus of $3 \times 10^{-2} \mathrm{~Pa}$ compatible with that predicted from eq 2.6. Between 1 and $0.01 \mathrm{~Hz}$ the system exhibits rheol ogical behavior with a square root dependence of the modulus on the frequency. Microrheological methods allow the investigation of higher frequency range, but the frequency range investigated to date does not allow the study of the elementary flexion modes. Indeed, present microrheol ogical experiments seem to fall close to the time scale near $\tau_{\mathrm{e}}$, where variation in the effective friction coefficient due to hydrodynamic interaction between a filament and the tube complicates the theoretical analysis of the experiments. Microrheological measurements are also probably influenced by nonlinear rheological effects due to the high velocity gradients around a small bead.

We note also that solutions of actin filaments are extremely sensitive to external forces. Stresses comparable to $0.1 \mathrm{~Pa}$ are sufficient to completely stretch filaments in regions under tension and buckle filaments in regions under compression. Buckling of filaments could be an effecient way of causing entanglements on a scale of $D$, leading to a possible work hardening of actin solutions via a mechanism similar to hernia formation seen in DNA electrophoresis.

\section{Discussion}

We have treated the dynamics of actin solutions as an equilibrium process. However actin filaments undergo a dynamic process of growth and depolymerization. Could this dynamic process be important for the rheology? Under the usual conditions the answer would seem to be no, except perhaps in the termination time for the plateau modulus. At dynamic equilibrium the critical concentrations for the two ends of an actin filament are different, leading to the process of treadmilling, 10 which implies a net loss of material at one end with a net gain of material at the other end. Thus each filament can be expected to advance though its tube at a constant speed. This propagative mode will be faster than the center of mass diffusion for sufficiently long filaments. One can estimate that for a filament of between 50 and $100 \mu \mathrm{m}$ length the process of tread-milling is as efficient as the process of reptation for the relaxation of stress. This, however, means experiments which take place on the scale of many hours. Agents which increase considerably the critical concentration could be expected to increase the speed of tread-milling and thus change the balance between these two processes, leading to a novel rheological regime for constraint release at long times. We note that there are here some anal ogies to the properties of living polymers where novel constraint release mechanisms al so exist. ${ }^{27}$ However systems of living polymers are still intrinsically equilibrium systems rather than stationary dynamic systems and are thus not entirely analogous. Finally, we have performed all our calculations in a regime where breakage due to external strain is rare, it is well-known that actin solutions are very sensitive to large shear rates. ${ }^{11}$

Acknowledgment. We would like to thank M.-F. Carlier, T. Duke, S. Leibler, J . Kaes, and E. Sackmann for discussions and suggestions made during this work. H.I. was supported by the DRET as a scientifique du contingent. A.M. would like to thank the hospitality of the University of Princeton where this work was completed.

\section{References and Notes}

(1) Korn, D. K.; Carlier, M-F.; Pantaloni, D. Science 238, 1987, 638.

(2) Gittes, F.; Mickey, B.; Nettleton, J .; Howard, J . J . Cell Biol. 120, 1993, 923-934.

(3) Ott, A.; Magnasco, M.; Simon, A.; Libchaber, A. Phys. Rev. E 1993, 48, 3, R1642.

(4) Takebayashi, T.; Morita, Y.; Oosawa, F. Nature 307, 1994, 58.

(5) Schmidt, C. F.; Barman, M.; Isenberg, G.; Sackmann, E. Macromolecules 1991, 24, 3111.

(6) Piekenbrock, Th.; Sackmann, E. Biopolymers 32, 1992, 1471.

(7) Farge, E.; Maggs, A. C. Macromolecules 26, 1993, 5041.

(8) Kaes, J .; Strey, H.; Sackmann, E. Nature 1994, 368, 226.

(9) Sackmann, E.; Kaes, J .; Stey, H. Adv. Mater. 6, 1994, 507.

(10) Bray, D. Cell Movements; Garland Publishing Inc.: London, 1992.

(11) J anmey, P. A. Curr. Opinion Cell Biol. 31, 1991, 4.

(12) Ruddies, R.; Goldman, W. H.; I senberg, G.; Sackmann, E. Eur. Biophys. J . 1993, 22, 309.

(13) Muller, O.; Gaub, H.; Baerman, M.; Sackmann, E. Macromolecules 24, 1991, 3111.

(14) Ziemann, F.; Radler, J .; Sackmann, E. Biophys. J . 66, 1994, 2210.

(15) J anmey, P. A.; Hvidt, S.; Kaes, J .; Lerche, D.; Maggs, A. C.; Sackmann, E.; Schliwa, M.; Stossel, T. P. Preprint, 1994.

(16) Landau, L.; Lifshitz, E. M. Theory of Elasticity; Pergamon Press: Oxford, U.K., 1986.

(17) Huse, D.; Maggs, A. C.; Leibler, S. Europhys. Lett. 12, 1990, 19.

(18) Doi, M.; Edwards, S. F. The theory of polymer dynamics; Oxford University Press; Oxford, 1989.

(19) de Gennes, P. G. Scaling Concepts in Polymer Physics; Cornell University Press: Ithaca, NY, 1979.

(20) Rubinstein, M.; Colby, R. H.; Dobrynin, A. V. Preprint, 1994.

(21) Semenov, A. N. J . Chem. Soc., Faraday Trans 2, 1986, 317. We note, however, that this free volume approach to the calculation of the tube size has recently be shown to be inadequate 22,23 for the case of completely rigid molecules. Rather in this case of one finds that $D_{t} \sim D$.

(22) Mori, Y.; Ookubo, N.; Hayakawa, R.; Wada, Y.J . Polym. Sci., Polym. Phys. Ed. 20, 1982, 2111.

(23) Brazhnik, O. D.; Khokhlov, A. R. Proc. SPIE 1990, 1402 (USSR-CSFR J oint Seminar on Nonlinear Optics in Control, Diagnostics and Modeling of Physical Processes).

(24) Hearst, J . E.; Harris, R. A.; Beals, E. J . Chem. Phys. 45, 1966, 8, 3106.

(25) Tinland, B.; Maret, G.; Rinaudo, M. Macromolecules 23, 1990, 596.

(26) Teraoka, I.; Langley, K. H.; Karasz, F. E. Macromolecules 1992, 25, 6106.

(27) Cates, M. E.; Candau, S. J . J . Phys.: Condens. Matter 2, 1990, 6869.

MA946418X 